\title{
Multiple Solutions of a Nonlinear Boundary Layer Problem
}

\author{
S. Chen
}

\begin{abstract}
We study a mathematical model for large-scale ocean circulation. Under reasonable physical assumptions, the governing partial differential equations reduce to a nonlinear third order ordinary differential equation $\phi^{\prime \prime \prime}+\lambda\left(\phi \phi^{\prime \prime} .-\phi^{\prime 2}\right)+1-\phi=0$ with stress-free conditions $\phi(0)=\phi^{\prime \prime}(0)=0$ and boundary condition $\phi(\infty)=1$. We prove, by a topological shooting method, that in the range of the parameter $\lambda>(27 / 4)^{1 / 3}$ there are at least two solutions. These solutions can be used further to construct the whole flow pattern in the ocean.
\end{abstract}

Keywords: Boundary layer, shooting method, Gulf Stream

AMS subject classification: 35Q10, 76U05

\section{Introduction}

Let $\eta$ be the independent variable and ${ }^{\prime}=d / d \dot{\eta}$. We study the existence of solutions of the initial-boundary value problem

$$
\begin{aligned}
& \phi^{\prime \prime \prime}+\lambda\left(\phi \phi^{\prime \prime}-\phi^{\prime 2}\right)+1-\phi=0 \\
& \phi(0)=\phi^{\prime \prime}(0)=0 \text { and } \phi(\infty)=1 .
\end{aligned}
$$

The differential equation (1) arises in the theory of ocean circulation and was developed by Ierley and Ruehr [2]. It is a two-dimensional, one layer model for large-scale ocean circulation with emphasis on the Gulf Stream. Assuming that the steady state vorticity equation holds, considering the ocean basin as a rectangular region, and taking into account the east-west flow of the wind, they observe a boundary layer is formed on the westernmost edge of the ocean basin. In the boundary layer, they then introduce the similarity function $\phi$ and assume that the stream function $\psi$ is proportional to $\phi$. Subsequently, they use an appropriate matched asymptotic expansion procedure to arrive at the initial-boundary value problem (1) for $\phi$. The complete derivation of the problem (1) is given in [1: Chapter 2] or [2], which gives a survey up to 1989 on this important subject in physical oceanography. In the same paper [2], in addition to asymptotic analysis in the boundary layer, they also show numerically that for problem

(1) the following statements are true:

(i) If $\lambda>0$, then exactly two solutions exist.

(ii) If $\lambda=0$, then one solution exists.

S. Chen: Howard Univ., Dep. Math., Washington, DC 20059, USA 
(iii) If $-0.29657<\dot{\lambda}<0$, then there are exactly two solutions.

(iv) If $\lambda<-0.29657$, then no solution exists.

In the trivial case $\lambda=0$, problem (1) becomes linear and statement (ii) can be verified easily. Concerning statements (i) and (iii), Lu and Troy [3] have rigorously proved the existence of solutions of problem (1) when the parameter $\lambda$ is small. We summarize their results in the following

Theorem 1: There is an $\epsilon_{0}>0$ such that if $|\lambda|<\epsilon_{0}$, then problem (1) has at least one solution and these solutions $\phi$ have the following asymptotic behavior:

(v) $(\phi(\eta)-1) e^{\eta / 8} \rightarrow 0$ as $\eta \rightarrow \infty$.

(vi) $\left|\phi^{\prime}(\eta) e^{\eta / 8}\right| \leq 3$ and $\left|\phi^{\prime \prime}(\eta) e^{\eta / 8}\right| \leq 3$ for all $\eta \geq 0$.

(vii) If $\phi^{*}(\eta)$ is the solution of problem (1) when $\lambda=0$, then $\lim _{\lambda \rightarrow 0}\left|\phi(\eta)-\phi^{*}(\eta)\right|=$ 0 uniformly in $\eta$.

For large negative $\lambda$ (cäse (iv)), Lu and Troy in [3] established the following statement.

Theorem 2: If $\lambda<-(2)^{1 / 3}$, then problem (1) has no sulution.

In the case (i) for large positive $\lambda$, Troy in [6] showed the following statement.

Theorem 3: If $\lambda>(27 / 4)^{1 / 3}$, let $\sigma_{1}, \sigma_{2}$ be the two negative roots of $\sigma^{3}+\lambda \sigma^{2}-1=$ $0, \sigma_{2}<\sigma_{1}$. Then there is at least one solution $\phi$ of problem (1) with $\phi^{\prime}(0)<-\sigma_{1}$.

Motivated by numerical studies, it is the purpose of this paper to prove the existence of the second branch of the solutions of problem (1) when $\lambda>(27 / 4)^{1 / 3}$. The main theorem in this work and the Theorem 3 partially confirm the numerical result (i), since in our assertion the parameter $\lambda$ has to be greater than $(27 / 4)^{i / 3}$. The significance of existence of the solutions $\phi$, i.e., $\psi$, can be realized by using $\phi$ as an outer expansion to match interior expansion so that a complete flow pattern may be constructed to demonstrate relatively uniform flow in the interior and intensification on the western boundary of the ocean. The technical part of this analysis has been carried out in [2].

Main Theorem: If $\lambda>(27 / 4)^{1 / 3}$, let $\sigma_{1}, \sigma_{2}$ be the two negative roots of $\sigma^{3}+$ $\lambda \sigma^{2}-1=0, \sigma_{2}<\sigma_{1}$. Then, in addition to the solutions in Theorem 9 , there is a solution $\phi$ of problem (1) with $-\sigma_{1} \leq \phi^{\prime}(0)$.

The proof is given in Section 3. Section 2 contains a brief review of the physical background needed to understand the role played by problem (1) in ocean circulation.

\section{Phyșical background}

The source of the problem investigated in this paper is a two-dimensional, one-layer model of the large-scale ocean circulation with emphasis on the Gulf Stream. The balance of terms in the governing partial differential equations suggests the appearance of a sharp boundary layer in the vicinity of the western boundary. The simplest such model problem from which a one-dimensional equation can be derived is due to Stommel [5] and was the first dynamical explanation of "westward intensification," or what is commonly referred to as the Gulf Stream. Later a model due to Munk [4], which 
incorporates a different mechanism for dissipation, yields a fourth-order problem, and is the linearized version of the problem we consider here.

The problem (1) stems from the so-called barotropic quasigeostrophic potential vor. ticity equation. Let $\psi$ be the stream function, $\Delta$ the Laplacian, and $J$ the Jacobian operator in $x, y$ variable. In non-dimensional form, the steady-state potential vorticity equation can be written as $J(\psi, q)=W+\kappa \Delta q$, where $q=y+\gamma \Delta \psi$ is the vorticity, $W=-\cos (\pi y / 2)$, and $\kappa, \gamma$ are physical constants. Readers may recognize that the Jacobian represents nonlinear convection and the Laplacian the viscous drag. In largescale ocean's motion the crucial effect of the Coriolis force must be taken into account due to the earth's variable rotation. Consequently we expect that the relation between the vorticity $q$ and the stream function $\psi$ will be altered. The term $W$ is intended to model the wind forcing blowing steadily across a rectangular ocean basin, varying with latitude. Here a coordinate system positive $y$ towards north and positive $x$ towards east is used. The boundary conditions are

$$
\psi(1, y)=\psi(-1, y)=\psi(x, 1)=\psi(x,-1)=0
$$

and

$$
\frac{\partial^{2} \psi}{\partial x^{2}}(1, y)=\frac{\partial^{2} \psi}{\partial x^{2}}(-1, y)=\frac{\partial^{2} \psi}{\partial y^{2}}(1, y)=\frac{\partial^{2} \psi}{\partial y^{2}}(-1, y)=0
$$

The last four conditions are referred to as stress-free boundaries.

In the middle of the basin, far from the boundary, it is generally assumed that the interior solution $\psi_{I}$ of the equation is well approximated by $\partial \psi / \partial x \sim W$; hence $\psi_{I} \sim(1-x) \cos (\pi y / 2)$. The interior solution $\psi_{I}$ is known as the Sverdrup balance. To satisfy the boundary condition on the left side of the basin, a significant boundary layer forms, in which $x$ derivatives are substantially greater than the $y$ derivatives. Within the boundary layer, we neglect the contribution due to $W$, but through the simple Sverdrup balance. It is $W$ that determines the parametric $y$ dependence of the interior solution, and by matching, that of the boundary-layer solution as well.

To derive an approximate one-dimensional description of this; we expand the solution about $y=-1$ by using a Taylor expansion of the wind-stress term. We neglect the $y$ derivatives except in evaluation of the Jacobian, which would otherwise vanish. Taking $\psi \sim \pi \phi(x)(y+1)$, we find

$$
\kappa \gamma \phi^{\prime \prime \prime \prime}=\pi \gamma\left(\phi^{\prime} \phi^{\prime \prime}-\phi \phi^{\prime \prime \prime}\right)+\phi^{\prime}
$$

Of particular interest is the limit of vanishing viscosity $\kappa \rightarrow 0$. The factor $\gamma$ is also small, about $10^{-3}$; hence in terms of $\eta=(\kappa \gamma)^{-1 / 3} x$, for asymptotically matching the interior solution we require

$$
\psi(\eta \rightarrow \infty, y \sim-1)=\pi \phi(\eta \rightarrow \infty)(1+y)=\psi_{I}(x \rightarrow-1, \dot{y} \sim-1)=\pi(\dot{1}+y)
$$

Therefore $\phi(\infty)=1$. Although the boundary layer equation is fourth order, it can be integrated once. Letting $\lambda=\pi\left(\gamma / \kappa^{2}\right)^{1 / 3}$ and using the stress-free and boundary conditions, we arrive at problem (1) (see [1: Chapter 2] or [2] for details). 


\section{Proof of the main theorem}

For convenience we make the transformation of the dependent variable $\phi=u+1$. Thus the equation and initial-boundary conditions for $u$ are

$$
\begin{aligned}
& u^{\prime \prime \prime}+\lambda u^{\prime \prime}-u+\lambda\left(u u^{\prime \prime}-\left(u^{\prime}\right)^{2}\right)=0, \\
& u(0)=-1, \quad u^{\prime \prime}(0)=0, \quad \text { and } u(\infty)=0 .
\end{aligned}
$$

Since the algebraic equation $\sigma^{3}+\lambda \sigma^{2}-1=0$ is closely associated with the differential equation (2), we state the following property whose proof is elementary.

Lemma 1: If $\lambda>(27 / 4)^{1 / 3}$, then the equation $\sigma^{3}+\lambda \sigma^{2}-1=0$ has three real distinct roots, $\sigma_{2}<\sigma_{1}<0<\sigma_{0}$, and $0<\sigma^{3}+\lambda \sigma^{2}-1$ for $\sigma_{2}<\sigma<\sigma_{1}$.

The idea of the proof of the main theorem is to use a topological shooting method by dropping the condition $u(\infty)=0$ in (2) and seeking an appropriate value of $u^{\prime}(0)$ so that the resulting initial value problem has a solution in $[0, \infty)$ and satisfies the boundary condition $u(\infty)=0$ at infinity. To proceed, for each $\beta$, let $v$ be the solution of the initial value problem

$$
\begin{aligned}
& v^{\prime \prime \prime}+\lambda v^{\prime \prime}-v+\lambda\left(v v^{\prime \prime}-\left(v^{\prime}\right)^{2}\right)=0 \\
& v(0)=-1, \quad v^{\prime}(0)=\beta, \quad v^{\prime \prime}(0)=0 .
\end{aligned}
$$

For each $\beta$, by the local existence theorem, there is a $\eta_{\beta}>0$ such that $v(\eta)<0$ is a solution of problem (3) and (4) in $\left(0, \eta_{\beta}\right)$. Therefore the function $h(\eta)=v^{\prime} / v$ is well defined in $\left[0, \eta_{\beta}\right)$ and $h(0)=-\beta, h^{\prime}(0)=-\beta^{2}$. Among many values of $\beta$, we define the shooting set

$$
A=\left\{\beta>-\sigma_{1} \mid h\left(\eta_{\beta}\right)=\sigma_{2} \text { and } h^{\prime}(\eta)<0 \quad\left(0 \leq \eta \leq \eta_{\beta}\right) \text { for some } \eta_{\beta}>0\right\} .
$$

Lemma 2: The set $A$ is non-empty, open, bounded below and above with $-\sigma_{1} \leq$ $\alpha=\inf A<-\sigma_{2}$.

Proof: If $v$ is the solution of (3) with $v(0)=-1, v^{\prime}(0)=-\sigma_{2}$ and $v^{\prime \prime}(0)=0$; we have $h(0)=\sigma_{2}$ and $h^{\prime}(0)=-\sigma_{2}^{2}<0$. Thus $h\left(\eta^{*}\right)<\sigma_{2}$ for some $\eta^{*}>0$ and $h^{\prime}(\eta)<0$ for all $\eta \in\left[0, \eta^{*}\right)$. Since the solutions of problem (3) continuously depend on initial values, $\sigma_{2}-\epsilon \in A$ for a small $\epsilon>0$. The fact $A$ is open can be verified by, again, continuity. If $-\sigma_{2} \in A$, by the definition of $A$, we have $h(0)=\sigma_{2}=h\left(\eta^{* *}\right)$ for some $\eta^{* *}>0$ and $h^{\prime}(\eta)<0$ for all $0 \leq \eta \leq \eta^{* *}$. This is not possible by mean value theorem. It is clear that any number $\beta>-\sigma_{2}$ does not belong to the set $A$

Now let $w$ be the solution of the particular shooting $w^{\prime}(0)=\alpha$, namely,

$$
\begin{aligned}
& w^{\prime \prime \prime}+\lambda w^{\prime \prime}-w+\lambda\left(w w^{\prime \prime}-\left(w^{\prime}\right)^{2}\right)=0 \\
& w(0)=-1, \quad w^{\prime}(0)=\alpha, \quad w^{\prime \prime}(0)=0 .
\end{aligned}
$$

Whenever $w(\eta) \neq 0$, we define $g(\eta)=w^{\prime} / w$. Then according to (5), we find that $g$ is a solution of the problem

$$
\begin{aligned}
& g^{\prime \prime}+(3 g+\lambda+\lambda w) g^{\prime}+g^{3}+\lambda g^{2}-1=0 \\
& g(0)=-\alpha, \quad g^{\prime}(0)=-\alpha^{2} .
\end{aligned}
$$

The proof of the main theorem is completed in the following lemma. 
Lemma 3: The solution $w$ of problem (5) can be continued to $[0, \infty)$ and $w(\infty)=0$. Consequently, $w$ is a solution of problem (2) with $-\sigma_{1} \leq w^{\prime}(0)$.

Proof: By the local existence theorem, let $\left[0, \eta_{0}\right)$ be the maximal interval of existence of problem (5). First we prove that $w \neq 0$ for all $\eta \in\left[0, \eta_{0}\right)$. Suppose not, let $\eta_{1}$ be the first zero of $w$. Therefore $g$ is well defined throughout $\left[0, \eta_{1}\right)$. By (6), we can say that initially $g$ satisfies $\sigma_{2}<g(\eta) \leq-\alpha \leq \sigma_{1}$ and is decreasing. We claim that $g$ is always decreasing in $\left[0, \eta_{1}\right)$. Suppose not, let $\eta_{2}$ be the first $\eta$ such that $g^{\prime}\left(\eta_{2}\right)=0$. If $g\left(\eta_{2}\right)^{\prime}<\sigma_{2}$, by continuity, there would be a $\beta \in A$ and $\beta<\alpha$, contradicting the definition of $\alpha$. If $g\left(\eta_{2}\right)=\sigma_{2}$, then both $g$ and the constant function $\bar{g}=\sigma_{2}$ are the solutions to the problem

$$
\begin{gathered}
g^{\prime \prime}+(3 g+\lambda+\lambda w) g^{\prime}+g^{3}+\lambda g^{2}-1=0 \\
g\left(\eta_{2}\right)=\sigma_{2}, \quad g^{\prime}\left(\eta_{2}\right)=0 .
\end{gathered}
$$

But $\bar{g}(0)=\sigma_{2} \neq g(0)=-\alpha$. This can not happen by uniqueness. Therefore $\sigma_{2}<$ $g(\eta) \leq-\alpha \leq \sigma_{1}$ for all $\eta \in\left[0, \eta_{2}\right]$. Since $\sigma_{2}<g\left(\eta_{2}\right)<\sigma_{1}$, evaluating (7) at $\eta_{2}$ and using Lemma 1 we conclude that $g^{\prime \prime}\left(\eta_{2}\right)<0$. Thus $g\left(\eta_{2}\right)$ is a local maximum. On the other hand, Lemma 1 also implies that $-\left(g^{3}+\lambda g^{2}-1\right) \leq 0$ for all $\eta \in\left[0, \eta_{2}\right]$. Since $g^{\prime}$ satisfies the first order differential equation

$$
\left(g^{\prime}\right)^{\prime}+(3 g+\lambda+\lambda w) g^{\prime}=-\left(g^{3}+\lambda g^{2}-1\right)
$$

and the initial condition $g^{\prime}(0)=-\alpha^{2}<0$, we find that $g$ is non-increasing in $\eta \in\left[0, \eta_{2}\right]$. This contradiction leads us to the facts that

$$
g^{\prime}(\eta)<0 \text { and } \sigma_{2}<g(\eta) \leq-\alpha \leq \sigma_{1} \quad \text { for all } \eta \in\left\{0, \eta_{1}\right) .
$$

Hence, in $\left[0, \eta_{1}\right)$, we have

$$
\sigma_{2}<g(\eta)=\frac{w^{\prime}(\eta)}{w(\eta)} \leq-\alpha .
$$

Integrating this once shows that $w$ is strictly bounded away from zero as $\eta$ goes to $\eta_{1}$. Thus $w(\eta) \neq 0$ for all $\eta \in\left[0, \eta_{0}\right)$. Repeating this argument in $\left[0, \eta_{0}\right)$ yields that $w(\eta)$ is bounded away from zero as $\eta$ goes to $\eta_{0}$ and inequality

$$
\sigma_{2}<g(\eta)=\frac{w^{\prime}(\eta)}{w(\eta)} \leq-\alpha
$$

now is valid for all $\eta \in\left[0, \eta_{0}\right]$. It results in that $w^{\prime}(\eta)$ is bounded as $\eta$ goes to $\eta_{0}$. Because $w^{\prime \prime}$ satisfies

$$
\left(w^{\prime \prime}\right)^{\prime}+\lambda(1+w) w^{\prime \prime}=w+\lambda\left(w^{\prime}\right)^{2} \quad \text { with } w^{\prime \prime}(0)=0
$$

a formula for $w^{\prime \prime}(\eta)$ shows that $w^{\prime \prime}(\eta)$ is bounded as $\eta$ goes to $\eta_{0}$. In conclusion, $w$ can be extended to $[0, \infty)$ and is never zero. Furthermore, we obtain

$$
\sigma_{2}<\frac{w^{\prime}(\eta)}{w(\eta)} \leq-\alpha \quad \text { for all } \eta \in[0, \infty) .
$$

This inequality implies that $w(\infty)=0$ 


\section{References}

[1] Hendershott, M.C.: Single layer models of the general circulation. In: The general circulation of the ocean (eds.: H. Abarbanel and W.R. Young and W.R. YOUNG). BerlinHeidelberg-New York: Springer - Verlag 1986.

[2] Ierle, G.R. and O.G. Ruehr: Analytic and numerical solutions of a nonlinear boundary layer problem. Stud. Appl. Math. 75 (1986), 1 - 36.

[3] Lu, C. and W.C. Trou: The behavior of solutions of a nonlinear boundary layer equation. In: Proceedings of the Microprogram on Nonlinear Diffusion Equations and Their Equilibrium States II (eds.: W.-M. Ni, L.A. Peletier and J. Serrin). Berlin-Heidelberg-New York: Springer - Verlag 1988.

[4] Munk, W.H.: On the uind-driven circulation. J. Meteorol. 7 (1950), 79 - 93.

[5] Stommel, H.: The westward intensification of wind-driven ocean currents. Trans. Amer. Geophys. Union 99 (1948), 202 - 209.

[6] Troy, W.C.: Solutions of a nonlinear boundary layer problem arising in physical oceanography. Rocky Mountain J. Math. 22 (1991), 813 - 820.

Received 27.11.1992; in revised form 24.08.1993 